RBMC

ISSN 2447-9071

doi 10.36414/rbmc.v6i14.33
Contato para correspondência: Hermínio Maurício da Rocha Sobrinho

E-mail:

herminio.sobrinho@gmail.com

Conflito de interesse: Não

Financiamento: Recursos próprios

Recebido: 12/01/2020

Aprovado: 03/02/2020

\title{
Neuroinflamação na doença de Alzheimer
}

\section{Neuroinflamation in Alzheimer's disease}

Annelisa Pimentel Rezende Machado'; Izabella Oliveira Carvalho' ${ }^{1}$ Hermínio Maurício da Rocha Sobrinho ${ }^{1,2}$

${ }^{1}$ Pontifícia Universidade Católica de Goiás - PUC Goiás

2 Universidade Estadual de Goiás - UEG

\section{Resumo}

A doença de Alzheimer (DA) é umas das principais demências do mundo, sendo caracterizada como uma desordem progressiva e crônica que leva a destruição de neurônios colinérgicos. O quadro clínico envolve uma série de eventos graduais desde a perda de memória episódica até o desfecho de deterioração da memória, do comportamento e da execução de movimentos. Este artigo teve como objetivo abordar o papel da neuroinflamação na patogênese da doença de Alzheimer, com ênfase na participação da imunidade inata no processo de neurodegeneração. Trata-se de uma revisão bibliográfica narrativa realizada no período de 2008 a 2019. As bases de dados para busca foram dados eletrônica Pubmed/MedLinee Portal Capes, utilizando-seos seguintes descritores "AlzheimerDisease, Inflammation, Neuroinflammation, Immunology, Cytokines". Foram encontradas 79 referências, 19 estudos foram excluídos por não obedecerem aos critérios de inclusão do trabalho. A patogenia da $D A$ associa-se à formação cerebral das placas senis, que são depósitos extracelulares do peptídeo $\beta$ amiloide eemaranhados neurofibrilares intracelulares constituídos, principalmente, por proteína Tau hiperfosforilada. Astrócitos e micróglias ativados são, caracteristicamente, encontrados próximos às placas senis, evidenciado a participação de elementos do sistema imune na neuroinflamação e neurodegeneração. Vários são os fatores capazes de ativar as vias inflamatórias na DA, como mutações genéticas, condições ambientais, emocionais, idadee hábitos de vida. Estes tipos deestímulos induzem um processo cíclico em que células daimunidadeinata como a micrógliaeo astrócitoliberammoléculas pró-inflamatórias como citocinas, radicais livres, neurotoxinas criando assim um estado inflamatório crônico, contribuindo para a disfunção e morte neuronal com consequente progressão da DA.

Palavras-Chave: Neuroinflamação, Resposta imune, Doença de Alzheimer.

\begin{abstract}
Alzheimer's disease (AD) is one of the world's leading dementias and is characterized as a progressive and chronic disorder that leads to the destruction of cholinergic neurons. The clinical picture involves a series of gradual events ranging from episodic memory loss to the outcome of memory impairment, behaviorandmovementperformance. This paperaimed to demonstrate the role of neuroinflammation in the pathogenesis of Alzheimer's disease, with emphasis on the participation of innate immunity in the neurodegeneration process. This is a narrative literature review conducted from 2008 to 2019. The search databases were electronic Pubmed / MedLine and Portal Capes, using the following descriptors "Alzheimer Disease, Inflammation, Neuroinflammation, Immunology, Cytokines". We found 79 references, 19 studies were excluded because they did notmeet the inclusion criteria. Thepathogenesis of $A D$ is associated with the brain formation of senile plaques, which are extracellular deposits of the amyloid $\beta$ peptide and intracellular neurofibrillary tangles consisting mainly of hyperphosphorylated tau protein. Astrocytes and activated microglia are characteristically found near the senile plaques, evidencing the participation of elements of the immune system in neuroinflammation and neurodegeneration. There are several factors that can activate the inflammatory pathways in $A D$, such as genetic mutations, environmental, emotional conditions, age and lifestyle. From such stimuli there is a cyclic process in which innate immunity cells such as microglia and astrocyte release proinflamma-
\end{abstract}


torymolecules such as cytokines, free radicals, neurotoxins thus creating a chronic inflammatory state contributing to neuronal dysfunction and death with consequent progression of $A D$.

Keywords: Neuroinflammation, Immune response, Alzheimer's disease.

\section{Introdução}

A doença de Alzheimer (DA) é uma desordem progressiva e crônica, caracterizada pela destruição de neurônios colinérgicos, sendo uma das principais causas de demência no mundo'. A idade é o fator de risco mais relevante no desenvolvimento dessa doença, apresentando uma prevalência de $10 \%$ em indivíduos acima de 65 anos, a qual aumenta para $40 \%$ naqueles com mais de 80 anos $^{2}$. A perspectiva para o futuro é de que a doença atinja mais de 80 milhões de pessoas em 2040, ao passo que, apenas no Brasil, o acometimento da população em 2020 será de $13 \%{ }^{3}$.

A sintomatologia desta doença envolve uma série de eventos graduais, começando com perda de memória episódica e podendo chegar até deterioração da memória, do comportamento e da execução de movimentos, com dependência permanente de cuidados familiares ${ }^{4}$. $O$ diagnóstico é clínico, utilizando como critérios o início do declínio das habilidades acima citadas, e o tempo de evolução da doença. O uso de exames de neuroimagem, como a ressonância magnética, auxilia na confirmação diagnóstica ${ }^{5}$. Atualmente, não há tratamentos que interrompam ou retardem a progressão da doença, e as terapias farmacológicas disponíveis aos pacientes apenas fornecem alívio sintomático ${ }^{6}$.

A DA é caracterizada, patologicamente, por acúmulo de proteínas deformadas no Sistema Nervoso Central (SNC): beta amiloide extracelular e proteína tau associada a microtúbulos intracelulares (MAPT). Essas inclusões levam à atrofia cerebral severa e neurodegeneração no hipocampo e no córtex cerebral ${ }^{7}$. Recentemente, há evidências da neuroinflamação, mediada por células imunes inatas cerebrais, na patogenia desta doença induzindo o processo de neurodegeneração ocasionando alterações funcionais e/ou morte neuronal ${ }^{8}$.

Portanto, abordar mecanismos imunológicos inflamatórios que podem estar envolvidos na patogênese desta doença é fundamental para despertar o estudo de novas terapias capazes de modular as vias moleculares envolvidas no desenvolvimento desta e também uma abordagem terapêutica mais eficaz para a DA. Diante disso, o objetivo do presente trabalho foi revisar o papel de mecanismos inflamatórios envolvidos na patogenia da e descrever as evidências da sua relação com o processo de neurodegeneração e progressão da doença.

\section{Métodos}

Trata-se de uma revisão bibliográfica narrativa, utilizando-se a base de dados eletrônica Pubmed/MeLine (US National Library of Medicine) e Portal Capes. Foram utilizados descritores em língua portuguesa e inglesa, especialmente os Descritores em Ciências da Saúde (DeCS): Alzheimer Disease, Inflammation, Neuroinflammation, Immunology, Cytokines. Foram avaliados e selecionados artigos publicados no período de 2008 a 2019. Foram encontradas 79 referências, deste total foram excluídas 19 referências que não apresentaram conteúdos que contribuíram para o cumprimento dos objetivos deste estudo, os trabalhos publicados antes do ano de 2008 e aqueles que não estavam disponíveis na íntegra nas bases de dados pesquisadas. Um total de 60 referências foram analisadas neste estudo.

\section{Resultados e Discussão}

A patogenia da DA está associada à formação cerebral das placas senis, que são depósitos extracelulares do peptídeo $\beta$ amiloide $(\beta A P)$ e emaranhados neurofibrilares intracelulares constituídos principalmente por proteína tau hiperfosforilada. Astrócitos e micróglias ativados são caracteristicamente encontrados próximos às placas senis, evidenciado a participação de elementos do sistema imune na neuroinflamação e neurodegeneração nesta doença. A hiperfosforilação da proteína TAU leva à desestruturação dos microtúbulos, gerando emaranhados neurofibrilares intracelulares9. Somado a isso, o acúmulo de peptídeo beta amiloide depositados em placas senis compõe a hipótese da cascata amiloidal, uma das bem mais aceitas como explicação para o estado inflamatório crônico. Uma teoria paralela é a colinérgica, a qual baseia-se na perda massiva de neurônios colinérgicos e, consequentemente, de colina acetiltransferase e de acetilcolinesterase, o que produz uma disfunção cognitiva ${ }^{10,11}$.

A DA tem como característica preponderante a existência de duas formas, sendo elas a familiar e a idiopática. A primeira é responsável por apenas $5 \%$ dos casos, envolve a manifestação precoce antes dos 65 anos e relaciona-se com mutações nos genes proteína precursora amiloide (APP), presenilina-1 (PSEN-1) e presenilina-2 (PSEN-2) 12. Já o subtipo idiopático corresponde a outra parcela de casos, e associa-se principalmente à idade e ao alelo E4 da apoliproteina E (APOE4), transportadora de colesterol ${ }^{12}$. 
Outro gene associado ao déficit cognitivo é o TREM-2 (Triggering Receptor Expressed on Myeloid cells-2), cuja variante R67H modifica sua ação neuroprotetora, aumentando em 3 vezes o risco de apresentar a comorbidade ${ }^{13}$. Estudos em roedores com envelhecimento acelerado e com inibição do TREM-2, demonstraram prejuízo da cognição e maior expressão de moléculas pró-inflamatórias, como fator de necrose tumoral (TNF- a) e interleucina 6 (IL-6) ${ }^{14}$.

Outra grande influência no desenvolvimento da DA são as alterações epigenéticas, que associam a competência dos fatores ambientais de impulsionarem mudanças no fenótipo do indivíduo sem a alteração da sequência de DNA ${ }^{15}$. Dentre as modificações epigenéticas que sensibilizam o fenótipo, as primordiais são: transformação de histonas e cromatinas, regulação do RNA não codificante e metilação do DNA. Os estímulos ambientais que levam a essa situação podem ser referentes à exposição a substâncias tóxicas ou metais pesados como alumínio, cobre e chumbo, à prática ou não de exercícios físicos ou até à uma mudança no comportamento a nível emocional ${ }^{16}$. O consumo de tabaco caracteriza-se como um risco significativo para a etiopatogenia da DA, uma vez que existe perda no número de receptores acetilcolina nicotina (nAChR) aumentando o metabolismo colinérgico no SNC. A ilustração sobre a etiologia e as formas clínicas da doença são apresentadas nas figuras 1 e 2 , a seguir.

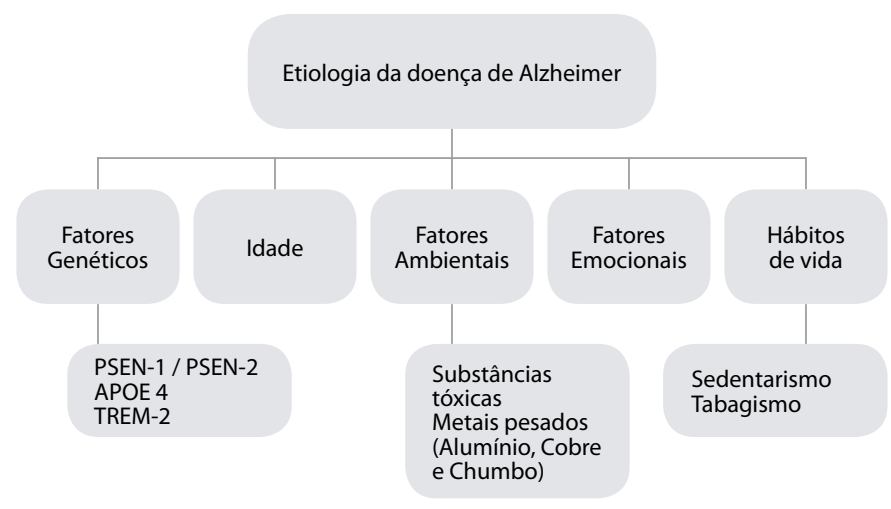

Figura 1. Fatores etiológicos da doença de Alzheimer. Legenda: PSEN-1/PSEN-2: presenilina 1 e 2; APOE-4: alelo E4 da apoliproteina $E ;$ TREM-2: triggering receptor expressed on myeloid cells- 2 .

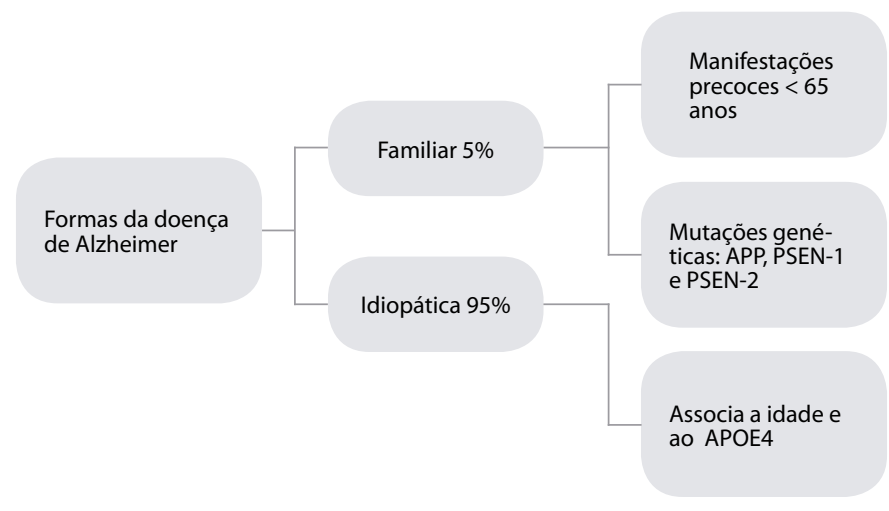

Figura 2. Formas da doença de Alzheimer. Legenda: PSEN-1/ PSEN-2: presenilina 1 e 2; APOE-4: alelo E4 da apoliproteina E.

\section{O papel do Sistema Imune na patogenia da doença de}

\section{Alzheimer}

O sistema imune é responsável pelo constante equilíbrio entre agressões teciduais e defesas, desencadeando respostas rápidas e a longo prazo afim de manter a integridade e funcionalidade dos tecidos e órgãos. A defesa contra agentes agressores teciduais é mediada pelas reações iniciais da imunidade inata e pelas respostas imunes tardias da imunidade adaptativa ${ }^{17}$.

A imunidade inata ou natural confere a primeira linha de defesa do organismo contra agentes agressores teciduais. Dentre os componentes da imunidade inata incluem: as barreiras físico-químicas (epitélios e agentes antimicrobianos), células apresentadoras de antígenos (APCs) e fagócitos (células dendríticas, macrófagos, monócitos e neutrófilos), células Natural Killer (NK), mastócitos, basófilos e eosinófilos. E ainda moléculas plasmáticas efetoras como as proteínas do sistema complemento, proteínas de fase aguda e citocinas ${ }^{18}$. Os componentes exclusivos da imunidade adaptativa são células denominadas linfócitos e seus produtos secretados, tais como anticorpos e citocinas. A ativação da resposta imune se desencadeia a partir de padrões moleculares associados a patógenos (PAMPs) compartilhados por patógenos microbianos, os quais são reconhecidos por receptores de reconhecimento de antígenos presentes na superfície de diversos tipos de leucócitos, especialmente pelas células apresentadoras de antígenos (APCS) ${ }^{19}$.

A inflamação é uma resposta imune inata projetada para proteger o hospedeiro de patógenos e agentes nocivos tissulares. Entretanto, a ativação do sistema imune e inflamação desencadeadas por processos de lesão e destruição celular no Sistema Nervoso Central, provavelmente devido a deposição 
de peptídeos beta-amiloide, desempenham um papel notável na patogênese da doença de Alzheimer ${ }^{19}$. Células do sistema imune inato residentes no cérebro, tais como a Micróglia e os Astrócitos, expressam receptores de reconhecimento de padrões (PRRs) capazes de reconhecer substâncias nocivas para os tecidos tais como: PAMPs (Pathogen-Associated Molecular Patterns) e DAMPs (Damage-Associated Molecular Patterns) que induzem ativação imunológica e neuroinflamação ${ }^{19,20}$.

A micróglia e os astrócitos expressam diferentes tipos de receptores similares a Toll (TRLs), grupo de receptores transmembrânicos expressos nas células da imunidade inata essenciais para o reconhecimento de moléculas associadas a patógenos e de substâncias endógenas produzidas ou liberadas em situações de dano tecidual, fundamentais na modulação da resposta inflamatória ${ }^{20}$. Evidências crescentes sugerem que proteínas desdobradas e agregadas, no cérebro, se ligam a receptores de reconhecimento de padrões na micróglia e astrócitos e desencadeiam uma resposta imune inata, caracterizada pela liberação de mediadores inflamatórios e neuroinflamação, que contribuem para a progressão e gravidade da doença de Alzheimer ${ }^{21}$.

Outro volumoso grupo de moléculas de intercomunicação e sinalização celular são as citocinas, as quais podem ser produzidas a partir da ativação de micróglias e astrócitos no sistema nervoso central (SNC) contribuindo diretamente para o processo de neuroinflamação. Posteriormente a uma exposição de agregados $\beta$-Amiloide ( $\beta A)$, a micróglia produz citocinas próinflamatórias como as Interleucinas (IL-1B, IL-6, IL-12) e o Fator de Necrose Tumoral alfa (TNF- $a)^{22}$.

Somado a estes fatores influentes na patologia da DA, o estresse oxidativo resultante da produção de intermediários reativos de oxigênio (ROIs) e espécies reativas de nitrogênio (ERN) desempenha papel fundamental na neuroinflamação. A produção abundante de radicais livres gera danos ao sistema de membranas neuronais com consequente peroxidação de lipídeos e oxidação das proteínas membranares ${ }^{21,22}$.

Apesar de ser a imunidade inata a mais comumente associada a DA, pesquisas também demonstram o envolvimento, em menor grau, da imunidade adaptativa na patogênese da doença. Aparentemente há uma diminuição, a nível periférico, dos linfócitos $T$, fenômeno que foi observado no sangue de alguns pacientes com $\mathrm{DA}^{23}$.

O papel das respostas imunes na patogenia da doença de Alzheimer ressaltando-se os mecanismos de ação e consequências patológicas está resumido e ilustrado na tabela 1 , logo a seguir.
Tabela 1. O papel das respostas imunes inata e adaptativa na patogenia da doença de Alzheimer.

\begin{tabular}{|c|c|c|c|}
\hline $\begin{array}{l}\text { Elemento } \\
\text { imonológico }\end{array}$ & $\begin{array}{l}\text { Mecanismo de } \\
\text { Ação }\end{array}$ & $\begin{array}{l}\text { Consequências } \\
\text { patológicas }\end{array}$ & Referências \\
\hline \multicolumn{4}{|l|}{ Imunidade Inata } \\
\hline Células Dendríticas & $\begin{array}{l}\text { Conexão entre as } \\
\text { imunidades inata e } \\
\text { adaptativa como de } \\
\text { célula apresentadora } \\
\text { de antígeno e pro- } \\
\text { dução de citocinas } \\
\text { pró-inflamatórias. }\end{array}$ & $\begin{array}{l}\text { Conexão entre as } \\
\text { imunidades inata e } \\
\text { adaptativa como de } \\
\text { célula apresentadora } \\
\text { de antígeno e pro- } \\
\text { dução de citocinas } \\
\text { pró-inflamatórias. }\end{array}$ & 24,25 \\
\hline Monócitos & $\begin{array}{l}\text { Diferenciam em } \\
\text { macrófagos/ } \\
\text { micróglia,fagocitam } \\
\text { restos teciduais, } \\
\text { produção de ROls } \\
\text { e citocinas pró- } \\
\text {-inflamatórias. }\end{array}$ & $\begin{array}{l}\text { Liberam espécies } \\
\text { reativas de oxigênio } \\
\text { (EROs) e Óxido } \\
\text { Nítrico durante a } \\
\text { fagocitose. }\end{array}$ & 24,25 \\
\hline Micróglia & $\begin{array}{l}\text { Reconhecimento de } \\
\text { sinais de perigo no } \\
\text { SNC. Regulação da } \\
\text { homeostase no SNC. } \\
\text { Resposta aguda local } \\
\text { no SNC mediada por } \\
\text { moléculas inflama- } \\
\text { tórias. }\end{array}$ & $\begin{array}{l}\text { Estimula a ativa- } \\
\text { ção da cascata do } \\
\text { complemento com } \\
\text { consequente dano } \\
\text { celular. O fenótipo } \\
\text { ativado libera subs- } \\
\text { tâncias neurotóxicas } \\
\text { e citocinas pró-infla- } \\
\text { matórias no SNC. }\end{array}$ & 26,27 \\
\hline Astrócitos & $\begin{array}{l}\text { Reconhecimento, } \\
\text { degradação e depu- } \\
\text { ração de peptídeos } \\
\text { beta amiloide. }\end{array}$ & $\begin{array}{l}\text { Produção de fatores } \\
\text { pró-inflamatórios: } \\
\text { interleucina, leu- } \\
\text { cotrieno, fator de } \\
\text { complemento e } \\
\text { proteases. }\end{array}$ & 24,25 \\
\hline \multicolumn{4}{|c|}{ Imunidade Adaptativa } \\
\hline $\begin{array}{l}\text { Linfócitos B/ } \\
\text { Plasmócitos }\end{array}$ & $\begin{array}{l}\text { Peptídeos } \beta \text { amiloide } \\
\text { estimulam as células } \\
\text { B a produzirem } \\
\text { anticorpos anti- } \beta \\
\text { amiloide. }\end{array}$ & $\begin{array}{l}\text { Anticorpos anti- } \beta \\
\text { amiloide ativam } \\
\text { células da glia de- } \\
\text { sencadeando uma } \\
\text { resposta imune. }\end{array}$ & 26,29 \\
\hline Linfócitos T CD4+ & $\begin{array}{l}\text { Migração para o } \\
\text { cérebro, levando } \\
\text { à interação com } \\
\text { células locais e } \\
\text { reconhecimento de } \\
\text { antígenos peptídicos } \\
\text { via MHC II. }\end{array}$ & $\begin{array}{l}\text { Células T CD4+ indu- } \\
\text { zem a diferenciação } \\
\text { de subtipos distintos } \\
\text { de micróglia, ex- } \\
\text { pressão de MHCII } \\
\text { influencia células } \\
\text { T a minimizarem os } \\
\text { danos da DA. }\end{array}$ & 30,31 \\
\hline Linfócitos T CD8+ & $\begin{array}{l}\text { Interação com } \\
\text { subtipos específi- } \\
\text { cos de micróglias } \\
\text { associadas a placa } \\
\text { de beta amiloide, } \\
\text { reconhecimento de } \\
\text { antígenos peptídicos } \\
\text { via MHC I. }\end{array}$ & $\begin{array}{l}\text { Modula a resposta da } \\
\text { microglia frente aos } \\
\text { depósitos de beta } \\
\text { amiloide. }\end{array}$ & 32,33 \\
\hline
\end{tabular}

O papel da Micróglia, Astrócitos e dos Mediadores Próinflamatórios na Neuroinflamação

As micróglias são células com alta capacidade de fagocitose presente no SNC, variando entre três formas de acordo com a necessidade local. Sua forma ameboide permite livre movimentação no tecido nervoso, porém sem induzir inflamação 
local, apenas age na modelação neuronal, sendo mais prevalente na formação do SNC. Outra configuração é a ramificada, esta já está em maior quantidade em cérebros adultos, sendo responsável por manter o equilíbrio imunológico neste ${ }^{34}$.

Sua terceira conformação é a micróglia ativa, que tem alta habilidade em fagocitose e apresentação de antígenos. Este fenótipo celular produz fatores pró-inflamatórios como citocinas, radicais livres, neurotoxinas podendo estimular a deposição de proteínas no SNC e provocar disfunção do sistema imune $^{35}$. Em resposta aos estímulos inflamatórios, a micróglia ativa libera mais moléculas inflamatórias, como TNF-a, IL-1 e óxido nítrico, levando a um circuito neuroinflamatório. A ativação dessa célula da glia pode ocorrer devido diversas condições, como lesões cerebrais, infecções, agregação de peptídeos e quaisquer outros elementos que podem causar danos ao SNC denominados, recentemente, como padrões moleculares associados a perigo ${ }^{36}$. O processo de ativação das micróglias está ilustrado, a seguir, na figura 3.

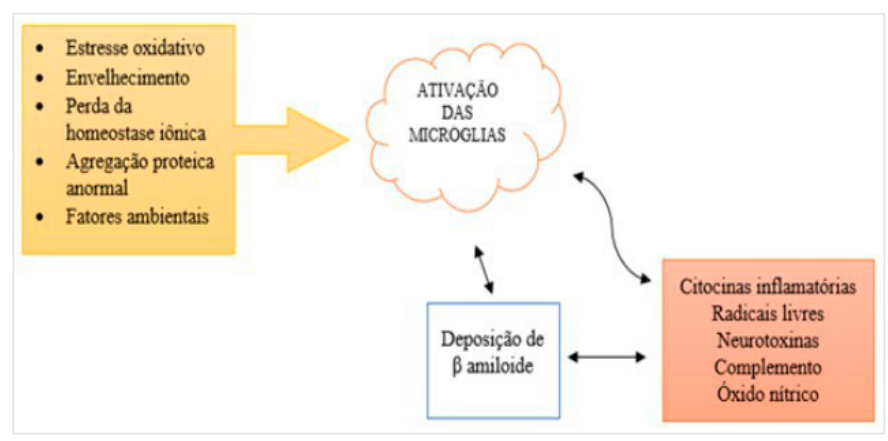

Figura 3. Processo de ativação das micróglias na doença de Alzheimer.

Outra célula da glia, o astrócito, participa na manutenção do equilíbrio cerebral. Esta é reconhecida pela captura de glutamato, evitando assim o acúmulo desse neurotransmissor e consequente neurotoxicidade ${ }^{37}$. Além disso, os astrócitos são responsáveis por conduzir as micróglias para áreas onde são necessárias. A ativação crônica ou desregulada da micróglia em indivíduo portador de doença neurodegenerativa pode levarà produção de mediadores pró-inflamatórios e diversas espécies reativas de oxigênio (EROs) que atingem o tecido nervoso do SNC provocando neurodegeneração. Dessa forma, a partir das lesões oxidativas e da redução do metabolismo energético, relaciona-se a potencialização da disfunção e morte neuronal com a formação de EROs no desenvolvimento da demência ${ }^{38}$.

Entre as principais moléculas inflamatórias associadas à patogenia da DA estão as citocinas IL-1a, IL-1 $\beta$, IL-6 e TNF- $\alpha$.
A IL-1 $\beta$, em particular, é uma das principais, colaborando para a evolução da DA devido ao estímulo para a produção de peptídeo $\beta$ amiloide neurotóxico, o aumento da fosforilação de proteína tau, a ativação de mais micróglias e astrócitos, liberando maior quantidade de citocinas, além de estimular a síntese de óxido nítrico39. Dessa forma, essas moléculas têm relevância primordial na formação de um ambiente nocivo no SNC, contribuindo para a neurodegeneração.

\section{Mecanismos da Neuroinflamação e Neurodegeneração}

A neuroinflamação ocorre quando há dano tecidual causado principalmente por compostos neurotóxicos, o que gera um conjunto de reações dinâmicas e complexas com consequências protetoras e nocivas ${ }^{40}$. Os mecanismos que contribuem para neuroinflamação e neurodegeneração são diversos, compondo assim um cenário multifatorial para o estado inflamatório na DA. Várias proteínas que surgem durante a neurodegeneração, incluindo $\beta$-amiloide, tau, proteínas de choque térmico e cromogranina, entre outras, agem como padrões moleculares associados ao perigo que, mediante $o$ envolvimento de receptores de reconhecimento de padrões, induzem vias de sinalização inflamatória e, por fim, levam à produção e liberação de mediadores capazes de provocar lesões teciduais e neurodegeneração ${ }^{41}$.

O peptídeo $\beta$ amilóide $(A \beta)$ resulta da clivagem de aminoácidos por meio da sua proteína percussora APP. Diversos estudam buscam esclarecer a função deste subproduto em indivíduos normais a fim de correlacionar suas modificações nas síndromes demenciais. Evidências recentes detectaram o $A \beta$ como parte da resposta imune inata sugerindo a deposição deste peptídeo como secundária a uma infecção vigente no organismo do indivíduo ${ }^{42}$. Dentre os patógenos virais capazes de regularem positivamente a geração de $A \beta$ destaca-se o vírus herpes simples 1 (HSV1) encontrado em agregados as placas amiloidais no cérebro de pacientes com $\mathrm{DA}^{43}$.

Em pessoas saudáveis durante a atividade neuronal é possível encontrar $A \beta$ que são liberados das vesículas intracelulares no espaço extracelular e não são passíveis de danos neurológicos, sendo necessário para a plasticidade e memória44. Desta forma diferentes moduladores incluindo o local da clivagem de APP que podem gerar frações A $\beta$ neurotóxicas como o peptídeo $A \beta 1-16$ e mutações em genes codificadores de APP, a saber PSEN1 e PSEN2, são vias que aumentam a proporção de $A \beta$ no SNC contribuindo diretamente para a agregação característica da $\mathrm{DA}^{45}$.

Normalmente a micróglia participa na manutenção das sinapses no SNC, já na vigência de patógenos ou estímulos inflamatórios ela adquire sua forma ameboide reativa ${ }^{46}$. Em 
seu estado normal, a micróglia expressa todos os receptores similares a Toll (TLR) em baixa quantidade, porém, após ativação, a expressão destes receptores aumenta, ampliando a capacidade da micróglia em reconhecer e ser ativada pelos PAMPs ou DAMPs, gerando inflamação e, assim, exercendo sua função de imunovigilância no cérebro ${ }^{47}$. Um desequilíbrio fisiológico no SNC induz a sua ativação aumentando assim a capacidade fagocitária e a produção de citocinas inflamatórias. Essa transformação é evidente no envelhecimento e na neurodegeneração, onde estudos evidenciam a reatividade de micróglias e astrócitos a peptídeos $\beta$ amiloides e formação de placas $\beta$ amiloides e emaranhados neurofibrilares no $\mathrm{SNC}^{48}$.

Além dos TLR, já citados anteriormente, outros PRRs presentes na micróglia são os inflamassomos. Esses, em consequência à ativação das micróglias, estão envolvidos na ativação da enzima caspase 1, a qual é fundamental para a produção de IL-1B e IL-18 ${ }^{49}$. Os inflamassomos predominantes na degeneração nervosa são as proteínas NLR, em especial a NLRP3. Esta é ativada pelos $\beta A$ intracelulares, o que resulta na liberação de IL-1B, instaurando o quadro inflamatório ${ }^{50}$. Estudos demonstram maior expressão de NLRP3 e NLRP1 em monócitos de pacientes com DA, coincidente com níveis elevados de IL-1B, afirmando a importância dessas proteínas ${ }^{51}$.

A neuroinflamação no SNC acaba se resumindo em um ciclo vicioso, no qual os $\beta A$ estimulam a micróglia a partir de diversas vias, como a via dependente de fator nuclear kappa $B$ (NFkB) e a via MAPK (proteína cinase ativada por mitógenos), o que resulta na liberação de citocinas como TNF-alfa, IL-1B e IL-6. O estado inflamatório exacerbado estabelecido contribui para a disfunção e morte de células do $\mathrm{SNC}^{52}$. O possível papel da micróglia nos processos de neuroinflamação e neurodegeneração na DA é ilustrado, logo a seguir, na figura 4.

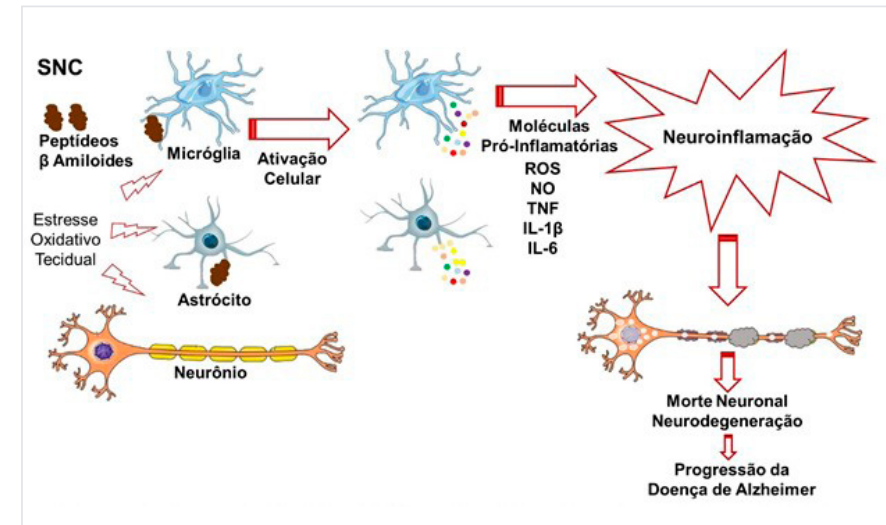

Figura 3. Processo de ativação das micróglias na doença de Alzheimer.

\section{Estratégias terapêuticas para modular a}

\section{Neuroinflamação na doença de Alzheimer}

A primeira estratégia terapêutica elaborada para a DA tem como mecanismo de ação a inibição da acetilcolinesterase (AChE). Esses medicamentos aumentam a quebra das APPs pela fração alfa da enzima secretase, o que gera fragmentos solúveis de $\beta A$, os quais são então fagocitados e eliminados pela micróglia. Dessa forma, o estímulo à alfa secretase diminui a deposição de $\beta A$ no $S_{N}{ }^{53}$. A medicação inicial com essa ação, lançada em 1993, foi a tacrina, que atualmente tem seu uso limitado devido à alta toxidade hepática. As drogas mais recentes no mercado para o tratamento da são a donepezil, galantamina, rivastigmina e memantina ${ }^{54}$.

Alguns estudos associam a progressão da DA com a ação da insulina no SNC, a qual aumenta a quebra da APP e a hiperfosforilação da proteína tau. Foi evidenciado ainda que beta amiloides estimulam a resistência a esse hormônio, aumentando os seus níveis séricos e formando um ciclo que acelera a neurodegeneração ${ }^{55}$. No entanto, a deficiência de insulina também apresenta influencia na DA, o que pode ser confirmado pelo fato de portadores tanto de diabetes tipo 1, quanto tipo 2, terem maiores chances de desenvolver a doença ${ }^{56}$. Dessa forma, cada vez mais estuda-se terapêuticas nessa linha de pensamento, como o uso de insulina intranasal nos casos de deficiência hormonal, e de agonistas do receptor do peptídeo 1 semelhante ao glucagon e metformina, para indivíduos com insulinorresistência ${ }^{57}$.

Outra área muito estudada é a de fitoquímicos, incluindo polifenois, cuja ação antioxidante e anti-inflamatória diminui a inflamação neuronal, contribuindo para uma melhor função $\operatorname{cognitiva}^{58}$. Esses compostos inibem a agregação de $\beta A$, a hiperfosforilação da proteína TAU e a produção de citocinas, sendo, portanto, agentes neuroprotetores. Alguns exemplos desses fitoquímicos são a cúrcuma, as catequinas, o licopeno, o resveratrol, blueberrys, chá verde e ginkgo biloba ${ }^{59}$.

Outros medicamentos que têm sido sugeridos em estudos são os anti-inflamatórios não esteroidais (AINEs), principalmente o ibuprofeno, diclofenaco e indometacina. Essas drogas diminuem o estado inflamatório no SNC a partir da inibição dasciclooxigenases (COX), além de inibirem as vias de ativação da micróglia ${ }^{60}$. Toda via, tem sido demonstrado que os AINEs diminuem o risco de desenvolver a doença, mas não são eficazes em atrasar o progresso dessa após instalada ${ }^{60}$.

\section{Conclusão}

Estudos evidenciaram que a patogenia da doença de Alzheimer envolve a deposição de proteína beta-amiloide e a hiperfosforilação da proteína Tau no SNC, as quais são potencial- 
izadas por estados inflamatórios (neuroinflamação) capazes de gerar neurodegeneração e progressão da doença. As principais células envolvidas neste processo são a micróglia e os astrócitos no SNC, que a partir da liberação de mediadores inflamatórios geram um processo cíclico de estímulo à neuroinflamação, levando a níveis cada vez maiores de lesão, degeneração e morte neuronal. Dessa forma, um maior entendimento sobre a etiopatogenia do processo de neuroinflamação é imperativo para que novas e mais eficazes opções terapêuticas sejam criadas.

\section{Referências}

1. Holtzman DM, John CM, Goate A. Alzheimer's disease: The challenge of the second century. SciTransl Med. 2011;3(77):77.

2. Neto HA (org.). A Disease Called Alzheimer. Mirabilia Medicinæ 09 (2017/2). V UNESC Medical Humanities SeminarV Seminário UNESC de Humanidades Médicas V Seminário UNESC de Humanidades Médicas Jul-Dez 2017/ISSN 1676-5818.

3. Korolev IO. Alzheimer's Disease: A Clinical and Basic Science Review. Medical Student Research Journal. 2014;4(1):24-33

4. Querfurth HW, LaFerla FM. Alzheimer's disease. N. Engl. J. Med. 2010;362:329-344.

5. Lane CA, Hardy J, Schott JM. Alzheimer's disease. Eur J Neurol. 2018;25(1);59-70.

6. Oboudiyat C, Glazer H, Seifan A, Greer C, Isaacson RS. Alzheirmer's disease. Semin Neurol. 2013;33(4):313-19.

7. Sereniki A, Vital MABF. A doença de Alzheimer: aspectos fisiopatológicos e farmacológicos. Rev Psiquiatr RS. 2008;30(1 Supl).

8. Takada LT. Imunidade inata e inflamação na patogênese da doença de Alzheimer. Arq. NeuroPsiquiatr. 2017;75(9).

9. Heppner FL, Ransohoff RM, Becher B. Immune attack: the role of inflammation in Alzheimer disease. Nat Rev Neurosci. 2015;16(6):358-72.

10. Heneka, MT et al. Neuroinflammation in Alzheimer's disease. Lancet Neurol. 2015;14(4):388-405.

11. Selkoe DJ, Hardy J. The amyloid hypothesis of Alzheimer's disease at 25 years. EMBO Mol Med. 2016;8(6):114.

12. Altmann A, Tian L, Henderson VW, Greicius MD. Sex modifies the APOE-related risk of developing Alzheimer disease. Ann Neurol. 2014;75:563-573.

13. Guerreiro R, Wojtas A, Bras J, Carrasquillo M, Rogaeva E, Majounie E, Cruchaga C, Sassi C, Kauwe JSK, Younkin $\mathrm{S}$, et al. TREM2 variants in Alzheimer's disease. $\mathrm{N}$ Engl Med. 2013;368(2):117-127.
14. Jiang T, Yu JT, Zhu XC, Tan MS, Gu LZ, Zhang YD, Tan L. Triggering receptor expressed on myeloid cells 2 knockdown exacerbates aging-related neuroinflammation and cognitive deficiency in senescence-accelerated mouse prone 8 mice. Neurobiol Aging. 2014;35(6):1243-51.

15. Adwan L, Zawia NH. Epigenetics: A novel therapeutic approach for the treatment of Alzheimer's disease. Pharmacology and Therapeutics. 2013;139(1):41-50.

16. Cacabelos R, Torrellas $C$. Epigenetics of aging and alzheimer's disease: Implications for pharmacogenomics and drug response. International Journal of Molecular Sciences. 2015;16(12):30483-30543.

17. Ransohoff RM, Brown MA. Innate immunity in the central nervous system. J Clin Invest. 2012;122(4):1164-71.

18. Heneka, MT, Golenbock DT, Latz E. Innate immunity in Alzheimer's disease. Nature Immunology. 2015;16(3):229-236.

19. Heneka MT, Kummer MP, Latz E. Innate immune activation inneurodegenerative disease. Nature Reviews Immunology. 2014;14:463-477

20. Trudler D, Farfara D, Frenkel D. Toll-like receptors expression signaling in glia cells in neuro-amyloidogenic diseases: Towards future therapeutic application. Mediators Inflamm. 2010:497987.

21. Gadoth N, Gobel HH. Oxidative Stress and Free Radical Damage in Neurology. Humana Press. 2011.

22. Couturier J, Page G, Morel M, Gontier C, Claude J, Pontcharraud R, Fauconneau B, Paccalin M. Inhibition of double-stranded RNA-dependent protein kinase strongly decreases cytokine production and release in peripheral blood mononuclear cells from patients with Alzheimer's disease. J Alzheimers Dis. 2010;21(4):1217-1231.

23. Schetters STT, Gomez-Nicola D, Garcia-Vallejo JJ, VanKooyk Y. Neuroinflammation: micróglia and T cells get ready to tango. Front Immunol. 2017;8:1905.

24. Kinoshita D. Alterações do sistema imunológico relacionadas ao envelhecimento e suas consequências. Revista da Universidade Ibirapuera - São Paulo. 2014;6:11-19

25. Bajramovic JJ. Regulation of innate immune responses in the central nervous system. CNS Neurol Disord Drug Targets. 2011;10(1):4-24

26. Gonçalves MV. Caracterização da resposta imune periférica na doença de Alzheimer. Dissertação de mestrado em Bioquímica, apresentada ao Departamento Ciências da Vida da Faculdade de Ciências e Tecnologia da Universidade de Coimbra. 2012. 
27. Tejera D, Heneka MT. Microglia in Alzheimer's disease: the good, the bad and the ugly. Curr Alzheimer Res. 2016;13(4):370-80.

28. Li C, Zhao R, Gao K, Wei Z, Yin MY, Lau LT, et al. Astrocytes: implications forneuroinflammatory pathogenesis of Alzheimer's disease. Curr Alzheimer Res. 2011;8(1): 67-80.

29. Bhamra MS, Ashton NJ. Finding a pathological diagnosis for Alzheimer's disease: areinflammatory molecules the answer? Electrophoresis. 2012;33(24):3598-607.

30. Andreasson KI, Bachstetter AD, Colonna M, Ginhoux F, Holmes C, Lamb B, et al. Targeting innate immunity for neurodegenerative disorders of the central nervous system. J. Neurochem. 2016;138:653-693.

31. Guillot-Sestier MV, Town T. Innate immunity in Alzheimer's disease: a complex affair. CNS Neurol Disord Drug Targets. 2013;12(5):593-607.

32. Unger MS, Marschallinger J, Kaindl J, Klein B, Johnson M, Khundakar AA, Rossner S, Heneka MT, Couillard-Despres $\mathrm{S}$, Rockenstein E, et al. Double cort inexpression in CD8+T-cells and microglia at sites of amyloid-beta plaques: A potential role in shaping plaque pathology? Alzheimers Dement. 2018;14(8):1022-37.

33. Marsh SE, Abud EM, Lakatos A, Karimzadeh A, Yeung ST, Davtyan $H$, et al. The adaptive immune system restrains Alzheimer's disease pathogenesis by modulating microglial function. Proc Natl Acad Sci USA. 2016;113:E1316-1325.

34. Cai Z, Hussain MD, Yan L-J. Microglia, neuroinflammation, and beta-amyloid protein in Alzheimer's disease. Int J Neurosci. 2014;124(5):307-321.

35. Kraft AD, Harry GJ. Features of microglia and neuroinflammation relevant to environmental exposure and neurotoxicity. Int J Environ Res Public Health 2011;8(7):2980-3018.

36. Colonna M, Butovsky O. Microglia function in the central nervous system during health and neurodegeneration. Annu. Rev. Immunol. 2017;35:441-68.

37. Barres BA. The mystery and magic of glia: a perspective on their roles in health and disease. Neuron. 2008;60(3):430-40.

38. Martin LJ. Biology of mitochondria in neurodegenerative diseases. Prog Mol Biol Transl Sci. 2012;107:355.

39. Dursun E, Gezen-AkD, Hanagası H, Bilgic, B, Lohmann E, Ertan S, et al. The interleukin 1 alpha, interleukin 1 beta, interleukin 6 and alpha-2-macroglobulin serum levels in patients with early or late on set Alzheimer's disease, mild cognitive impairment or Parkinson'sdisease. J
Neuroimmunol. 2015;15(283):50-7.

40. Payão SLM, Gonçalves GM, de Labio RW, Horiguchi L, Mizumoto I, Rasmussen LT, et al. Association of interleukin $1 \mathrm{~b}$ polymorphisms and haplotypes with Alzheimer's disease. J Neuroimmunol. 2012;247(12):59-62.

41. Eisele YS, Fritschi SK, HamaguchiT, Obermuller U, Fuger P, Skodras A et al. Multiple factors contribute to the peripheral induction of cerebral beta-amyloidosis. J Neurosci. 2014;34:10264-10273.

42. Bisht K, Sharmaa K, Tremblaya ME. Chronic stress as a risk factor for Alzheimer's disease: Roles of micróglia mediated synaptic remodeling, inflammation, and oxidative stress. Neurobiology of Stress. 2018;9:9-21.

43. Lim SL, Rodriguez-Ortiz CJ, Kitazawa M. Infection, systemic inflammation, and Alzheimer's disease. Microbes Infect. 2015;17(8):549-556.

44. Saido T, Leissring MA. Proteolytic degradation of amyloid beta-protein. Cold Spring Harb. Perspect. Med. 2012;2(6):a006379.

45. Barber RC. The genetics of Alzheimer's disease. Scientifica (Cairo). 2012;2466210.

46. Lannes N, Eppler E, Etemad S, Yotovski P, Filgueira L. Microglia at center stage: a comprehensive review about the versatile and unique residential macrophages of the central nervous system. Oncotarget. 2017;8(69):114393-413.

47. Sadigh-Eteghad S, Sabermarouf B, Majdi A, Talebi M, Farhoudi M, Mahmoudi J.Amyloid-beta: a crucial factor in Alzheimer's disease. Med PrincPract. 2015;24(1):1-10.

48. Ghosh S, Wu MD, Shaftel SS, Kyrkanides S, LaFerla FM, Olschowka JA, O'Banion MK. Sustained interleukin-1 over expression exacerbates tau pathology despite reduced amyloid burden in an Alzheimer's mouse model. J Neurosci. 2013;33(11):5053-64.

49. Latz E, Xiao TS, Stutz A. Activation and regulation of the inflammasomes. Nat. Rev. Immunol. 2013;13(6):397-411.

50. Cox DJ, Field RH, Williams DG, Baran M, Bowie AG, Cunningham $C$, Dunne $A$. DNA sensors are expressed in astrocytes and microglia in vitro and are upregulated during gliosis in neurodegenerative disease. Glia. 2015;63(5):812-25.

51. SaresellaM, La Rosa F, Piancone F, et al. The NLRP3 and NLRP1 inflammasomesare activatedin Alzheimer's disease. Mol. Neurodegener. 2016;11:23.

52. Koistinaho J, Malm T, Goldsteins G. Glycogensynthase kinase-3beta: a mediator of inflammation in Alzheimer's disease? Int J Alzheimers Dis 2011:129753. 
53. Mahdavi M, Saeedi M, Gholamnia L, Jeddi SAB, Sabourian $R$, Shafiee A, Foroumadi A, Akbarzadeh T. Synthesis of Novel Tacrine Analogs as Acetylcholinesterase Inhibitors. J. Heterocyclic Chem. 2017;54:384- 390.

54. Viegas FPD, Simões MCR, Rocha MD, Castelli MR, Moreira MS, ViegasJunior C. Doença de Alzheimer: Caracterização, Evolução e Implicações do Processo Neuroinflamatório. Rev. Virtual Quim. 2011;3(4):286-306.

55. Stanley M, Macauley S, Holtzman D. Changes in insulin and insulin signaling in Alzheimer's disease: cause or consequence? J. Exp. Med. 2016;213(8):1375-1385.

56. Sims-Robinson C, Kim B, Rosko A, Feldman E. How does diabetes accelerate Alzheimer disease pathology? Nat. Rev. - Neurol. 2011;6(10):551-559.

57. Chen Z, Zhong C. Decoding Alzheimer's disease from perturbed cerebral glucose metabolism: Implications for diagnostic and therapeutic strategies. Prog. Neurobiol. 2013; 108:21-43.

58. Thanthaeng N, Poulose SM, Miller MG, Shukitt-Hale B. Preserving brainfunction in aging: The anti-glycative potential of berry fruit. Neuromolecular Medicine. 2016;18(3):465-473.

59. Uribarri J, delCastillo MD, de laMaza MP, Filip R, Gugliucci A, Luevano-Contreras $C$, et al. Dietary advanced glycation end products and theirrole in health and disease. Advances in Nutrition. 2015;6(4):461-473.

60. Breitner JC, Baker LD, Montine TJ, Meinert CL, Lyketsos CG, Ashe KH, Brandt J, Craft S, Evans DE, Green RC, Ismail MS, Martin BK, Mullan MJ, Sabbagh M, Tariot PN, ADAPT Research Group. Extended results of the Alzheimer's diseaseanti-inflammatory prevention trial. Alzheimer's \& Dementia. 2011;7(4):402-411. 